\title{
Correction to: Computed Free Energies of Peptide Insertion into Bilayers are Independent of Computational Method
}

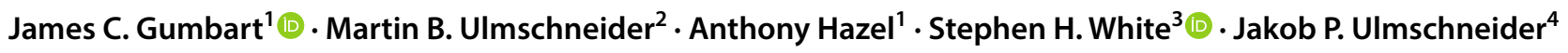

Published online: 25 June 2018

○) Springer Science+Business Media, LLC, part of Springer Nature 2018

Correction to: The Journal of Membrane Biology https://doi.org/10.1007/s00232-018-0026-y

The original version of the article unfortunately contained an error in NIH support grant number RO1-GM74639 in the Acknowledgements section. The correct grant number is RO1-GM74637. This has been corrected with this erratum.

The original article can be found online at https://doi.org/10.1007/ s00232-018-0026-y.

James C. Gumbart

gumbart@physics.gatech.edu

$\triangle$ Jakob P. Ulmschneider

jakob@sjtu.edu.cn

1 School of Physics, Georgia Tech, Atlanta, GA, USA

2 Department of Chemistry, King's College, London SE1 1DB, UK

3 Department of Physiology \& Biophysics, University of California at Irvine, Irvine, CA, USA

4 Department of Physics and the Institute of Natural Sciences, Shanghai Jiao Tong University, Shanghai 200240, China 